\title{
Das milde Auge des Gesetzgebers
}

\author{
Ökologisch-ethische Investments sind nicht nur für Anleger sondern auch für \\ die Politik attraktiv. Weltweit wurden Gesetze eingeführt, die die Berücksich- \\ tigung von ökologisch-ethischen Aspekten bei der Geldanlage fördern sollen - \\ allerdings nicht immer mit Erfolg.
}

A Von Thomas Loew uch die Politik versucht ökologischethische Investments (auch Socially Responsible Investments, SRI) zu fördern. Eine Fallstudie des Instituts für ökologische Wirtschaftsforschung zur Wirkung von Regulierungen auf ökologisch-ethisches Investieren hat drei SRIförderliche Regulierungen identifiziert (1):

- Die Offenlegungspflicht,

- Steuererleichterungen, und

- die Verpflichtung zur Berücksichtigung von ökologisch-ethischen Aspekten (2).

Das prominenteste Beispiel für die Offenlegungspflicht ist in Großbritannien anzutreffen. Alle betrieblichen Pensionsfonds sind verpflichtet in ihren schriftlich definierten Anlagepolitiken auch darüber Auskunft zu geben, ob und gegebenenfalls wie bei der Anlage der eingenommenen Beiträge ökologische oder ethische Kriterien berücksichtigt werden. Auch Pensionsfonds die keine SRI-Anlagepolitik verfolgen, müssen explizit darauf hinweisen. Die Offenlegungspflicht hat sich deutlich positiv auf den britischen SRI-Markt ausgewirkt und gleichzeitig international eine Vorbildrolle eingenommen. Inzwischen wurde die Offenlegungspflicht auch in Deutschland, Frankreich sowie Australien eingeführt, während sie in Belgien und Kanada noch diskutiert wird. Auf EUEbene sowie in Österreich und Neuseeland konnte die Offenlegungspflicht bislang politisch nicht durchgesetzt werden.

Allein die Existenz einer Offenlegungspflicht sagt jedoch nichts über ihre Bedeutung für den SRIMarkt aus, denn sie wird auf verschiedene Anlageformen angewendet, die länderspezifisch eine unterschiedliche Relevanz am jeweiligen Kapitalmarkt haben. In Großbritannien, Frankreich und in Deutschland wird die Offenlegungspflicht auf betriebliche Pensionsfonds angewendet. Pensionsfonds spielen am britischen Kapitalmarkt eine herausragende Rolle und ihre Vermögensbestände umfassen rund 90 Prozent des nationalen
Vereinzelt wird auch eine Verpflichtung zur Nachhaltigkeitsberichterstattung oder im Fall Deutschland auch das Energieeinspeisegesetz im Zusammenhang mit den oben dargestellten Regulierungen genannt. Diese werden jedoch als mittelbar förderliche Regulierungen betrachtet und nicht weiter betrachtet, weil sie sich nicht direkt auf die Förderung von ökologisch-ethischen Geldanlagen beziehen (Siehe Abbildung 1).

\section{Positive Wirkungen in Großbritannien}

Bruttoinlandprodukts (3). In Frankreich und Deutschland dagegen ist noch vergleichsweise wenig Kapital in Pensionsfonds angelegt. In Australien wird die Offenlegungspflicht nicht auf Pensionsfonds, sondern allgemein auf Versicherungen, Investmentfonds und andere Anlageformen angewendet. Auch hier ist also ein vergleichsweise großes Kapitalvolumen von der Offenlegungspflicht betroffen.

Steuererleichterungen für ökologisch-ethische Geldanlagen werden nur in den Niederlanden gewährt. Durch die so genannte Regeling Groenprojekten werden seit 1995 die Erträge aus Investmentfonds, die gezielt in niederländische Umweltschutz- oder Sozialprojekte investieren, steuerlich begünstigt. Inzwischen sind über 1,5 Milliarden Euro in diesen Fonds angelegt worden. In Schweden wurde die Berïcksichtigung von ökologisch-ethischen Aspekten für eine Reihe von (privat verwalteten) Reservefonds des staatlichen Rentensystems gesetzlich vorgeschrieben.

In Großbritannien hat sich die Offenlegungspflicht deutlich auf den dortigen Markt ausgewirkt. Nach einer bereits im Jahr 2000 durchgeführten Studie von Eugenie Mathieu gibt nur ein geringer Anteil (14 Prozent) der Pensionsfonds an, ökologisch-ethische Aspekte im Rahmen ihrer Anlageverwaltung in keinerlei Weise zu berücksichtigen (4). Allerdings ist der Anteil der Fonds, die systematisch anhand von definierten Positiv- oder Negativkriterien ihr Anlageuniversum nach ökologisch-ethischen Zielsetzungen ausrichten, mit acht Prozent ebenfalls gering. Die größte Gruppe (42 Prozent) umfasst Fonds, die in ihrer Anlagepolitik "nur" darauf verweisen, dass Auswirkungen von ökologisch-ethischen Fragestellungen auf die finanzielle Performance berücksichtigt werden sollen. Schließlich geben zwölf Prozent der Pensionsfonds an, dass sie eine Engagementstrategie verfolgen wollen. Das heißt, dass sie sich als Anteilseigner bei den Unternehmen, in die sie investiert haben, aktiv für eine nachhaltigere Unternehmenspolitik einsetzen wollen.

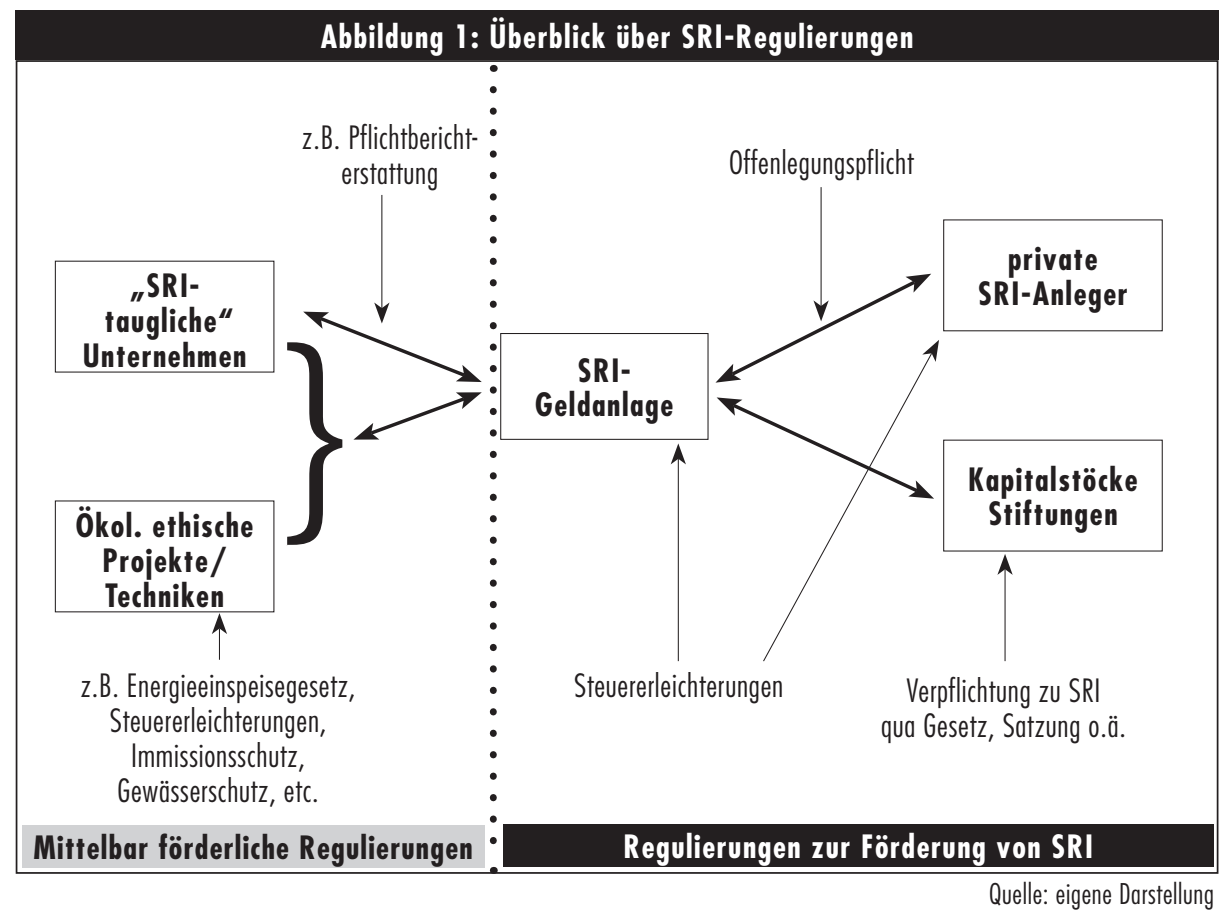


In diesem Zusammenhang muss berücksichtigt werden, dass bei der Studie zur Offenlegung nur die Ausrichtung der Strategie angegeben wurde. Mit welcher Intensität und Ernsthaftigkeit sie tatsächlich von den Pensionsfonds verfolgt wird, wurde nicht untersucht. Kritiker gehen davon aus, dass sich in den Fondsgesellschaften nur wenig verändert hat. Befürworter wie Sparkes weisen darauf hin, dass in vielen größeren Pensionsfonds eigene SRI-Abteilungen eingerichtet worden sind, und sehen entsprechend große Veränderungen (5).

\section{- Keine Wirkung in Deutschland}

Eine gänzlich andere Situation herrscht in Deutschland vor. Unter den rund 3.500 zugelassenen privaten Riesterrentenprodukten befinden sich nur 13 mit ökologisch-ethischer Ausrichtung (6). Dabei handelt es sich teilweise um Versicherungsprodukte, die bereits vor Inkrafttreten der Regulierung am Markt angeboten wurden. Die Anbieter waren alle schon vorher auf dem deutschen SRI-Markt aktiv. Die Offenlegungspflicht hat also keine positiven Auswirkungen auf den Markt der privaten Altersvorsorge. Für den offensichtlichen Misserfolg der Regelung dürfte die rechtliche Umsetzung der Offenlegungspflicht bei der privaten Riesterrente ausschlaggebend sein. Wenn Anbieter keine SRI-Politik im Rahmen der Anlage verfolgen, müssen sie darauf nur einmalig im "Kleingedruckten" bei Versicherungsabschluss hinweisen. Nur die Anbieter von Riesterrenten, die SRI in der Anlagepolitik verfolgen, sind verpflichtet ihren Kunden dazu jährlich zu berichten. Der ursprünglich beabsichtigte Anreizmechanismus wurde somit umgekehrt. Für die SRI-Anbieter entsteht in gewissem Umfang ein Mehraufwand, während die konventionellen Anbieter sich der Berichtspflicht durch einen einmaligen Hinweis entziehen können. Hier besteht noch politischer Handlungsbedarf.

Trotz des deutschen Fehlschlags ist die Offenlegungspflicht als ein vielversprechendes umweltpolitisches Instrument anzusehen. Sie dürfte sich weiter verbreiten und wie in Großbritannien einen positiven Einfluss auf die Entwicklung der SRI-Märkte haben.

\section{Anmerkungen}

(1) Die Studie wird im Rahmen des Forschungsvorhabens "Politikmuster der Entwicklung internationaler Märkte für Innovationen nachhaltigen Wirtschaftens - vom Pilotmarkt zum Lead-Markt", gefördert vom Bundesministerium für Bildung und Forschung (BMBF), durchgeführt, das von der Forschungsstelle für Umweltpolitik (Koordination), dem Institut für ökologische Wirtschaftsforschung, dem Deutschen Institut für Wirtschaftsforschung und dem Zentrum für Europä- ische Wirtschaftsforschung bearbeitet wird.

(2) Vgl. hierzu und zum Folgenden: Loew, Thomas: Internationale Entwicklung der Regulierungen zur Förderung ökologisch-ethischer Finanzdienstleistungen, Diskussionspapier des IÖW 56/02, Berlin 2002. Download unter www.ioew. de/home/downloaddateien/DP5602.pdf

(3) Döring, Diether, Die Zukunft der Alterssicherung, Frankfurt 2002, S. 92

(4) Mathieu, Eugenie: Response of UK Pension Funds to the SRI Disclosure Regulation, UKSIF 2000. Download unter www.uksif.org//publications/reprt-2000-10/ content.shtml (5) Vgl. Sparkes, Russell: Socially Responsible Investment A Global Revolution. Chichester 2002, S. $352 f f$

(6) Vgl. Germanwatch (Hrsg.): Übersicht Nachhaltigkeitsberichte zur "Riesterrente". Im Web unter www.germanwatch. org/rio/riester/index-Dateien/start.html

\section{Der Autor}

Thomas Loew ist wissenschaftlicher Mitarbeiter im Forschungsfeld ökologische Unternehmenspolitik des Instituts für ökologische Wirtschaftsforschung (IÖW).

Kontakt: IÖW, Potsdamer Str. 105, 10785 Berlin. Tel. 030-884594-17, E-Mail: Thomas.Loew@ioew.de

\section{Wie sag ich's meinen Kunden?}

\section{Nachhaltige, ethisch-ökologische oder Social Responsible Investments: Prinzipi- engeleitete Investments, wie sie von Experten genannt werden, stehen derzeit im Blickpunkt der Öffentlichkeit. Doch bislang zögern Bankberater bei der Ver- mittlung, Kunden beim Kauf der entsprechenden Anlagen. Abhilfe schafft da nur eins: angemessene Information.}

$\mathrm{V}$ Von Petra Thome iele Anleger assoziieren mit Nachhaltigkeit in erster Linie Umweltaspekte. Doch der Begriff reicht weiter. Nachhaltigkeit ist die Verknüpfung ökonomischer, ökologischer und sozialer Ziele. Anbieter grüner "Geldanlagen" investieren in Unternehmen, die nachhaltig wirtschaften. Mit anderen Worten: Neben der rein ökonomischen Gewinnmaximierung wird bei der nachhaltigen Investition die Verantwortung gegenüber Mensch, Umwelt und Gesellschaft gewährleistet.
In Deutschland öffneten kirchliche Organisationen den Markt für nachhaltige Geldanlagen. Sie suchten Ende der achtziger Jahre nach Möglichkeiten, ihr Geld nach ethischen und ökologischen Kriterien für sich arbeiten zu lassen. Als Reaktion auf den Wunsch nach religiös motiviertem Sparen legte SEB Invest 1989 den ersten deutschen ethisch-ökologischen Fonds, den SEB Invest ÖkoRent, auf. Doch der Absatz verlief anfangs schleppend, das Interesse war Anfang der neunziger Jahre gering. Vor allem Umweltschützer, Mit- glieder kirchlicher Organisationen oder sozial engagierte Personen legten ihre Gelder im Privatkundenbereich nachhaltig an. Heute findet die nachhaltige Geldanlage auch im klassischen Kundensegment zunehmend Akzeptanz. Auf der institutionellen Seite stieß und stößt der Gedanke der Nachhaltigkeit zumeist bei Kirchen, Gewerkschaften und Sozialen Einrichtungen auf ein stetig wachsendes Interesse.

Durch das gestiegene Interesse wird das Anlagesegment mittlerweile auch für Finanzdienstleister attraktiver. Waren zuerst nur kleinere oder ausländische Kapitalanlagegesellschaften, vornehmlich aus Österreich und der Schweiz, neben der SEB Invest auf dem deutschen Markt tätig, so bieten inzwischen auch die großen Investmenthäuser nachhaltige Investmentmöglichkeiten an. Das Interesse der Big Player ist ein untrügliches Zeichen für die Bedeutung, die Aktualität und die nachhaltigen Perspektiven dieser Art von Geldanlage.

\section{Stolperstein Komplexität}

Trotz der positiven Marktentwicklung haben prinzipiengeleitete Geldanlagen immer noch nicht den 
(c) 20I0 Authors; licensee IÖW and oekom verlag. This is an article distributed under the terms of the Creative Commons Attribution Non-Commercial No Derivates License (http://creativecommons.org/licenses/by-nc-nd/3.o/), which permits unrestricted use, distribution, and reproduction in any medium, provided the original work is properly cited. 